\section{AUTO-EVALUACIÓN PREVIA A LAS PRÁCTICAS DE LABORATORIO QUÍMICO: INTRODUCCIÓN AL AUTO- APRENDIZAJE}

\author{
Patricia Noguera Murray \\ Luis Antonio Tortajada Genaro \\ Julia Atienza Boronat

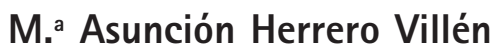 \\ Departamento de Química, Instituto de Reconocimiento Molecular \\ y Desarrollo Tecnológico \\ Universidad Politécnica de Valencia. Camino de Vera $s / n$ \\ pnoguera@qim.upv.es, luitorge@qim.upv.es \\ matien@qim.upv.es, maherrer@qim.upv.es
}

\section{SELF-EVALUATION TO CHEMISTRY LABORATORY SESSIONS: INTRODUCTION TO SELF-LEARNING}

ABSTRACT: In this work a strategy to improve learning outcomes of laboratory sessions of a Chemistry subject of the Bachelor's Degree in Rural and Agrifood Engineering is shown. In this experience the e-learning platform Poliformat was used to propose timed pre-lab questionnaires to allow self-assessment of students before laboratory sessions, this allowed an increase in the preparation and the interest of the student on the performance of the laboratory session. The effectiveness of this strategy was established from the analysis of data collected during its application and also by means of an opinion call. It is noteworthy that these questionnaires have induced change of attitude among students, encouraging an active posture (and positive) towards the laboratory session.

KEY WORDS: E-learning; Sakai; pre-lab questionnaires; assessment; self-learning.

\section{INTRODUCCIÓN}

Las clases prácticas de laboratorio son una parte muy importante de la docencia, ya que en ellas los alumnos pueden aprender conceptos teóricos y adquirir destrezas procedimentales y actitudinales. Por ejemplo, pueden adquirir conocimientos teóricos relacionados con la química (ilustrando ideas y conceptos, exponiendo teorías para verificar empíricamente), conocimientos prácticos (manipulación de equipos y productos químicos, aprender procedimientos de seguridad, medir de forma precisa, observar con cuidado),
RESUMEN: En este trabajo se muestra una estrategia para mejorar los resultados de aprendizaje en las prácticas de laboratorio en una asignatura de Química del Grado en Ingenieria Agroalimentaria y del Medio Rural realizando cuestionarios previos temporizados usando la plataforma e-learning PoliformaT. De este modo, se ha permitido la auto-evaluación de los alumnos antes de realizar una sesión de prácticas, fomentando la preparación y el interés del alumno en su realización y consiguiendo un mayor aprovechamiento de los recursos. La eficacia de esta estrategia se ha establecido a partir del análisis de datos recogidos durante la aplicación y realizando un sondeo de opinión. La aplicación de estos cuestionarios ha permitido un cambio de actitud en los alumnos, fomentando una actitud activa (y positiva) hacia las prácticas y consiguiendo un mayor aprovechamiento de las mismas.

PALABRAS CLAVE: E-learning; Sakai; cuestionarios previos; evaluación; auto-aprendizaje.

conocimientos científicos (aprender a observar, deducir e interpretar) y competencias generales (trabajo en equipo, realización de informes y presentaciones, participación en discusiones, manejo del tiempo, aprender a resolver problemas) (Reid y Shah, 2007). Por ello, los objetivos de la práctica deben estar fijados y desarrollados de forma clara y, además, deben ser conocidos por los alumnos (Reid y Shah, 2007).

Tradicionalmente, las prácticas de laboratorio de química comienzan con una pequeña presentación al inicio de la 
sesión en la que el profesor resume el experimento, sus objetivos e indica los procedimientos específicos directamente relacionados con el experimento a realizar, y los alumnos siguen el guión de prácticas como una receta. En muchos casos, el alumno sigue el procedimiento al pie de la letra, línea a línea e intentando conseguir el mismo dato que sus compañeros, sin tener una visión global del proceso que está realizando y no entendiendo cómo interpretar los resultados experimentales. Por otro lado, al carecer de una visión global de las prácticas, el alumno no se da cuenta de que en el laboratorio no sólo aprenden los conceptos químicos, también se les introduce en el método científico. Si a todo esto añadimos que hay alumnos que consideran que no va a trabajar en un laboratorio, la actitud de los mismos en las sesiones de laboratorio suele ser, en general, pasiva. Sin embargo, no toda la culpa la tiene el alumno, ya que hay que reconocer que éste recibe y debe procesar una ingente cantidad de información (Reid y Shah, 2007; Carnduff y Reid, 2003). En la Figura 1 se muestra la gran variedad de información que recibe el alumno y las relaciones que debe realizar.

El hecho de que algunos alumnos asistan a las prácticas de laboratorio con actitud pasiva e incluso rechazo, hace que el proceso de aprendizaje no se optimice y que los recursos se infrautilicen. Por ello, cualquier acción que repercuta en una mayor implicación de los alumnos, conseguirá facilitar su aprendizaje y evitar así el abandono. Una acción que puede incrementar el beneficio de las clases prácticas es realizar tareas pre-laboratorio en las que se traten con- ceptos o aspectos de la práctica en estudio, con ellas se consigue reducir la sobrecarga de información que éste recibe, permitiendo que la sesión se centre en lo importante. De este modo se aumenta el aprendizaje y se favorece la actitud positiva del alumno (Johnstone y Al-Ahuaili, 2001; Schmid y Yeung, 2008). Por otro lado, una buena preparación reduce la ansiedad y aumenta la confianza de los alumnos, lo que se traduce en una experiencia más productiva y un aprendizaje más positivo (McKelvy, 2008; Llorens-Molina, 2009).

En la bibliografia hay descritas una gran variedad de actividades pre-laboratorio (Reid y Shah, 2007; Burewicz y Miranowicz, 2006; McKelvy, 2008; Dalgarno et al., 2009; Limniou, Papadopoulos y Whitehead, 2009; Sierra et al., 2009). De entre ellas, una tarea que ha proporcionado excelentes resultados, y que es sencilla de aplicar, es la elaboración de cuestionarios que deben completarse antes de realizar la sesión de prácticas (Nicholls, 1999; Masson, 2001). En este sentido, las plataformas empleadas en la educación a distancia o e-learning pueden ser de gran utilidad (Yang y Tsai, 2010; Roffe, 2002) ya que permiten la inclusión de animaciones o gráficos. Sin embargo, y aunque los alumnos prefieren preguntas online animadas y/o interactivas a preguntas estáticas, la efectividad del aprendizaje no depende del formato de pregunta (Yeung et al., 2009). Realizar los cuestionarios pre-laboratorio con estas plataformas presentan a priori una serie de ventajas: el alumno controla su proceso de aprendizaje -ya que pueden completarlos en el momento y lugar que

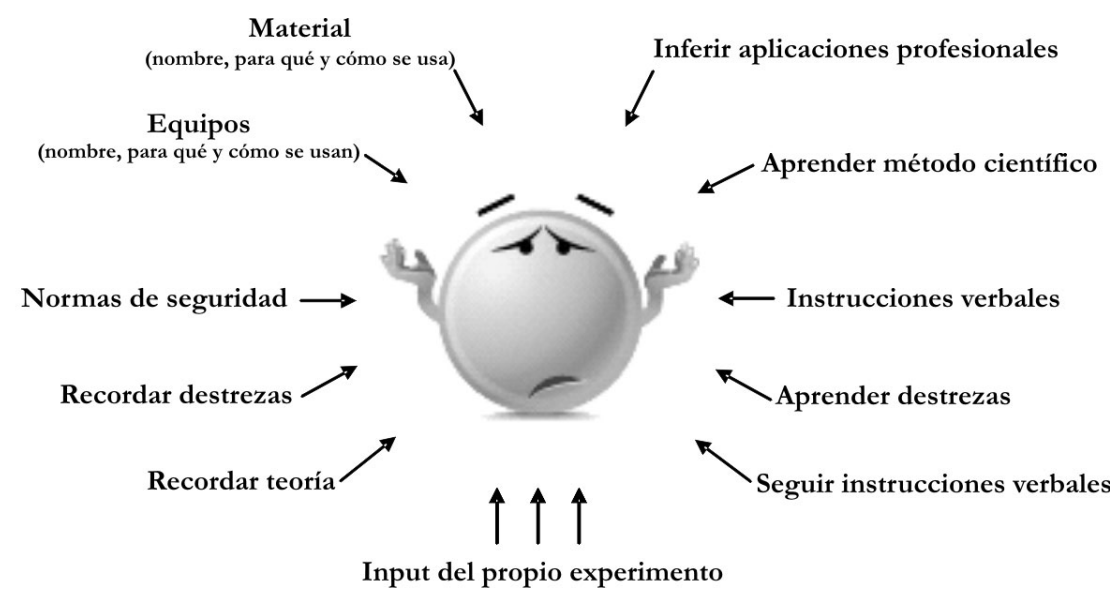

Figura 1. Fuentes de información de un alumno en un laboratorio de química. 
desee-, y se facilitan las tareas de corrección y de retroalimentación (Jenkins, 2004; Fike, Fike, Doyle y Connelly, 2010). Además, el alumno no está pasivo al interactuar con el ordenador.

Los cuestionarios previos pueden conseguir que se adquieran una parte de los objetivos de aprendizaje que se deben alcanzar con la práctica (Figura 2). Por ello, a la hora de diseñarlos no solo hay que definir los objetivos de aprendizaje; también hay que adaptar el manual de laboratorio y las preguntas post-laboratorio. Es decir, que para aplicar esta metodología, no es necesario cambiar drásticamente los experimentos que se realizan, solo hay que variar la forma en la que se desarrollan.

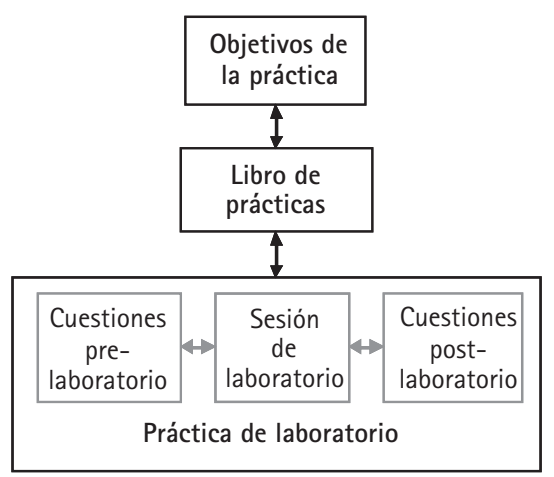

Figura 2. Actividades realizadas en las sesiones de laboratorio para alcanzar los objetivos de aprendizaje.

\section{Objetivos}

El objetivo general de este trabajo ha sido incorporar actividades pre-laboratorio de carácter no presencial con el fin de incrementar los conocimientos previos del alumno en las prácticas de laboratorio, de este modo se consigue una mayor implicación del alumno en su aprendizaje, y, por tanto, un mayor aprovechamiento de las sesiones de prácticas.

\section{Desarrollo}

La acción propuesta comenzó a aplicarse en el curso 201011 en un grupo de una asignatura de química impartida en primer curso del Grado en Ingeniería Agroalimentaria y del Medio Rural de la Escuela Técnica Superior de Ingeniería Agronómica y del Medio Natural de la Universidad Politécnica de Valencia, utilizando la plataforma docente de e-learning PoliformaT basada en el LMS (Learnig Management System) open source Sakai.

La incorporación de esta actividad a la docencia implicó realizar una etapa de planificación donde se elaboró un listado de conceptos teórico-prácticos considerados como prerrequisitos, se modificó el sistema de evaluación y se diseñaron los cuestionarios (Tortajada-Genaro et al., 2011). Finalmente, se evaluó globalmente la actividad con el fin de estimar la eficacia de la estrategia propuesta y realizar acciones para su mejora. Seguidamente se indican los resultados más destacados y como se plantea la actividad en el presente curso académico 2011-12.

\section{Resultados}

Cuestionarios pre-laboratorio. La premisa a la hora de plantear los cuestionarios es que un alumno debe contestar las preguntas con la información dada únicamente en el manual de prácticas (de ahí la necesidad de revisarlo y reeditarlo). Tras el primer año de aplicación se llegó a la conclusión de la necesidad de diseñar los cuestionarios (para cada una de las prácticas) con diferentes colecciones de preguntas. Una dedicado a la base teórica, otra al material de laboratorio específico o novedoso a utilizar, otra a la seguridad y tratamiento de los residuos generados, $y_{1}$ por último, otra a la realización de cálculos. Cada batería de cuestiones está formada por, al menos, 10 preguntas de entre las que se seleccionaba de forma aleatoria una o dos (en función de la práctica). El número total de preguntas planteadas oscilaba entre 4 y 8 , presentándose cada pregunta como una página Web diferente. En la Figura 3 se muestra un ejemplo de una de las cuestiones planteadas; la plataforma muestra al alumno el enunciado y el bloque al que pertenece la pregunta así como la figura asociada a la pregunta y de la que se debe extraer información. Además, se indica la puntuación asociada a la pregunta y el tiempo restante para completar el cuestionario.

Además, para minimizar el plagio entre alumnos se establece que las posibles respuestas se planteen de forma 


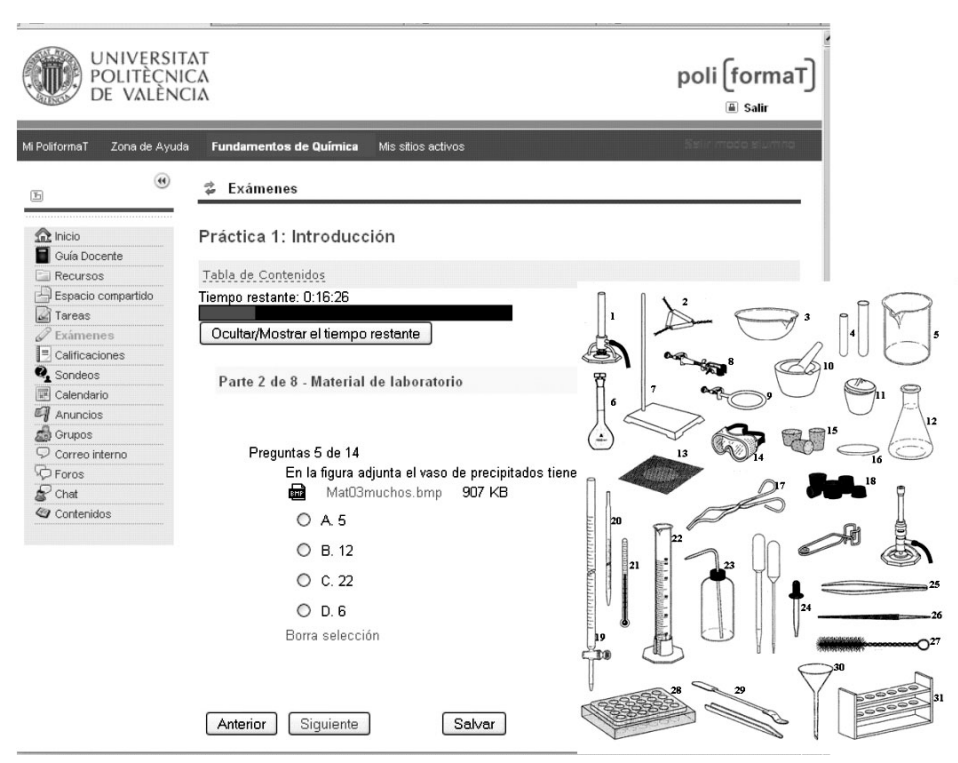

Figura 3. Ejemplo de cuestión planteada en el cuestionario pre-laboratorio.

aleatoria y que cada pregunta se muestre en una página Web diferente. El tiempo permitido para completar el cuestionario depende del número total de preguntas, con un máximo de 10 minutos. El objetivo es garantizar que los alumnos repasaran el material de preparación antes de rellenar el cuestionario, evitando una lectura superficial del mismo, buscando la respuesta concreta demandada. Un aspecto interesante de los cuestionarios, es que se permite que un alumno lo pueda repetir hasta tres veces guardándose la nota más alta, así se fomenta el interés por conseguir una mayor nota y se reduce la presión por la evaluación. Es de destacar que la plataforma PoliformaT facilita la gestión de los resultados ya que guarda para cada alumno el número de intentos realizados, la nota obtenida y el tiempo dedicado. Además, se pueden descargar los resultados individualizados por alumno o por pregunta, lo que permite detectar fallos comunes y abordarlos al inicio de la sesión, consiguiendo así una retroalimentación verdaderamente efectiva.

Libro de prácticas de laboratorio. Con el fin de que el alumno logre una mayor autonomía se ha actualizado el libro de prácticas. En este sentido se ha incluido una serie de recordatorios para evitar los errores procedimentales más comunes (Figura 4).
Evaluación. La incorporación de una nueva metodología a la docencia implica, necesariamente, una modificación del sistema de evaluación. Por ello, se consideró que asignando un $20 \%$ de la nota final de prácticas a esta actividad, se lograba la implicación de los alumnos más reticentes a realizar tareas.

Análisis de resultados. Los resultados preliminares han permitido estimar el impacto de la autoevaluación previa a las prácticas. De los 95 alumnos que estaban matriculados en el grupo, 62 participaron en esta actividad. La mayoría de los alumnos contestaron en un tiempo que oscilaba entre 2 y 4 minutos y obtuvieron calificaciones superiores a 7 , indicando que los alumnos han preparado la práctica. Por otra parte, se ha detectado un mayor aprovechamiento

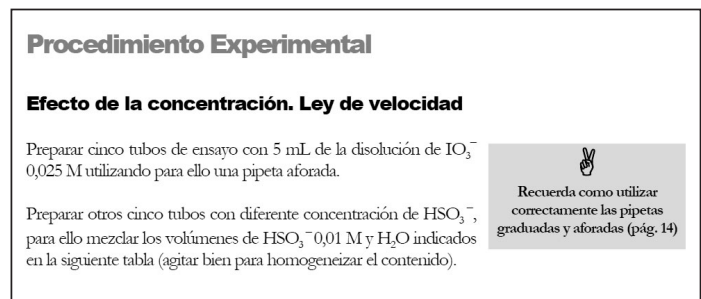

Figura 4. Ejemplo de un recordatorio.

doi: 10.3989/arbor.2011.Extra-3n3156 
del tiempo de prácticas, una reducción de errores y una mayor motivación, permitiendo una mayor interacción positiva entre alumno y profesor.

Sin considerar el tiempo dedicado a la creación de las preguntas, la introducción de esta actividad no implica un trabajo adicional para los profesores, ni se incrementa el tiempo en el laboratorio. Sin embargo, ha supuesto un aumento de, al menos, unas tres horas de trabajo extra al alumno, que lógicamente influye en el proceso de aprendizaje de los mismos. De hecho, los profesores que han aplicado esta metodología destacan que el cambio más notable observado es que los alumnos que han participado en esta actividad no realizan la práctica de forma mecánica, tienen un mayor interés, e incluso profundizan en aspectos que de otra forma no lo harían. Esto concuerda con lo observado por Nicholls (1999).

Para evaluar el grado de satisfacción de los alumnos y detectar posibles problemas surgidos durante el desarrollo de la actividad, se realizó un sondeo de opinión. Del análisis de las respuestas obtenidas se observa que el 100\% de los alumnos participantes consideraron que habian accedido a esta herramienta sin problemas y la mayoría (62\%) consideró que el nivel de las preguntas planteadas era fácil o muy fácil. El 70\% de ellos considera que el tiempo fijado para realizar el cuestionario es suficiente. Finalmente, la mitad de los alumnos consideran que la realización de los cuestionarios previos le ha facilitado la compresión de la misma. Es de destacar que, aunque hay alumnos que consi- deran que esta actividad no les ha facilitado la compresión de la práctica, estos mismos alumnos han dedicado menos tiempo a realizar la práctica que sus compañeros que no lo han hecho, aumentando por ello su efectividad.

Tareas de futuro. Con los datos del curso 2011-12 se pretende correlacionar la nota de prácticas global y de la asignatura con la obtenida en los cuestionarios, $y$, además, con parámetros que evalúan el interés del alumno (número de tests contestados, tiempo dedicado, etc.); de este modo, se podrán extraer conclusiones cuantitativas sobre la incorporación de estos cuestionarios en la actividad docente.

\section{ConClusión}

Se puede concluir que los beneficios obtenidos en la aplicación de esta metodología superan los inconvenientes de su desarrollo. De hecho los alumnos mejoraron su capacidad y destrezas en el laboratorio y consideraron que esta actividad ha facilitado la compresión de la práctica. Por otro lado, los profesores destacan el cambio de actitud observado en los alumnos que han participado. En resumen, la realización de una evaluación previa de los conocimientos de los alumnos, utilizando la plataforma PoliformaT, ha fomentado una actitud activa (y positiva) de los alumnos hacia las prácticas y ha conseguido un mayor aprovechamiento de las mismas.

\section{AGRADECIMIENTOS}

A la Universidad Politécnica de Valencia por la financiación del Proyecto de Innovación y Mejora Educativa (Ref. A011/10) concedida al grupo Trabajo y Evaluación de Asignaturas mediante Técnicas de Evaluación Continua Formativa (TECOF)

\section{REFERENCIAS}

Burewicz, A. y Miranowicz, N. (2006): "Effectiveness of multimedia laboratory ins- truction", Chemistry Education: Research and Practice, vol. 7, pp. 1-12.

Carnduff, J. y Reid, N. (2003): Enhancing undergraduate chemistry laboratories: pre-laboratory and post-laboratory exercises, Royal Society of Chemistry.

Dalgarno, B.; Bishop, A. G.; Adlong, W. y Bedgood, D. R. (2009): "Effectiveness of a virtual laboratory as a preparatory resource for distance education chemistry students", Computers \&t Education, vol. 53, pp. 853-865.

Fike, D. S.; Doyle, D. y Connelly, R. J. (2010): "On-line vs paper evaluations of fa- 
culty: When less is just as good", The Journal of Effective Teaching, vol. 2, pp. 42-54.

Jenkins, M. (2004): "Unfulfilled promise: Formative assessment using computer-aided assessment", Learning and Teaching in Higher Education, vol. 1, pp. 67-80.

Johnstone, H. y Al-Shuaili, A. (2001): "Learning in the laboratory; some thoughts from the literature", University Chemistry Education, vol. 5, pp. 42-51.

Limniou, M.; Papadopoulos, N. y Whitehead, C. (2009): "Integration of simulation into pre-laboratory chemical course: Computer cluster versus WebCT", Computers \& Education, vol. 52, pp. 45-52.

Llorens-Molina, J. A. (2009): "Design and assessment of an online prelab model in general chemistry: A case study", Journal of the Research Center for Educational Technology, vol. 4, pp. 15-31.

Masson, M. R. (2001): "Experience with a random questionnaire generator in the chemistry laboratory and for other continuous assessment", Uni- versity Chemistry Education, vol. 5, pp. 9-15.

McKelvy, G. M. (2008): "Preparing for the chemistry laboratory: An internet presentation and assessment tool", University Chemistry Education, vol. 2, pp. 46-49.

Nicholls, B. S. (1999): "Pre-Laboratory support using dedicated software", University Chemistry Education, vol. 3, pp. 22-27.

Reid, N. y Shah, I. (2007): "The role of laboratory work in university chemistry", Chemistry Education Research and Practice, vol. 8, pp. 172-185.

Roffe, I. (2002): "E-learning: engagement, enhancement and execution", Quality Assurance in Education, vol. 10, pp. 40-50.

Schmid, S. y Yeung, A. (2008): "The influence of a pre-laboratory work module on student performance in the first year chemistry laboratory", Research and Development in Higher Education, vol. 28, pp. 471-479.

Sierra, J.; Bosque, J.; García, A.; Blanc, R.; Del, M.; Gámiz, L. y Alés, F. (2009): "Aprendizaje de procesos analíticos mediante clases prácticas con labo- ratorios virtuales", Enseñanza de las Ciencias, Número Extra VIII Congreso Internacional sobre Investigación en Didáctica de las Ciencias, Barcelona, pp. 2466-2470.

Tortajada-Genaro, L. A.; Noguera, P.; Atienza, J.; Herrero, M. A., García-Rupérez, J.; Sanchís, P.; Vidal, B.; Fenollosa, M. L.; Ribal, F. J.; Bes, M. A.; Blasco, E. y Muñoz, M. J. (2011): Auto-evaluación previa a las Prácticas mediante la Plataforma de e-Learning PoliformaT. Una experiencia multidisciplinar, Segunda Conferencia Internacional en Fomento e Innovación con Nuevas Tecnologías en la Docencia de la Ingeniería (FINTDI), en prensa.

Yang, Y. F. y Tsai, C. C. (2010): "Conceptions and approaches to learning through online peer assessment", Learning and Instruction, vol. 20, pp. 72-83.

Yeung, A.; Schmid, S. A.; George, A. V. y King, M. (2009): "Increasing students' interest and motivation through effective design on online learning environments", Proceedings of the Motivating Science Undergraduates, A. Hugman (Ed.), Sydney, pp. 132138. 